\title{
XVIII. Der Albit von Rischuna in morphologischer Beziehung.
}

\author{
Von \\ Berta Krebs in Heidelherg.
}

(Mit 34 Textfiguren.)

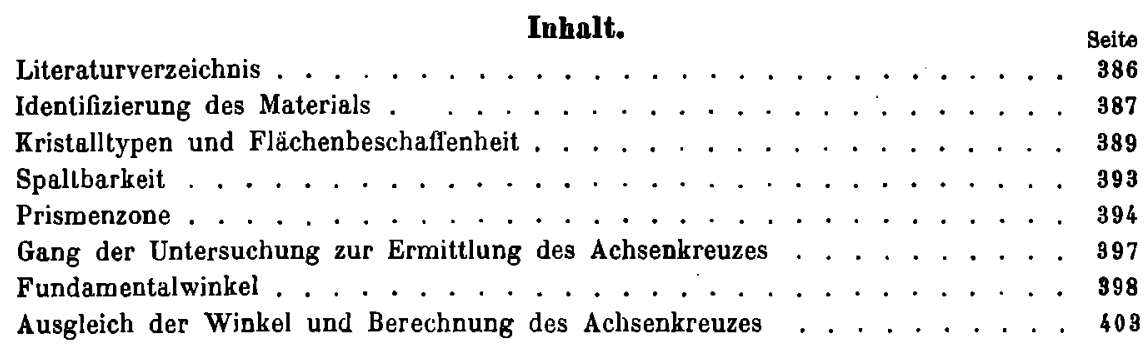

Literaturverzelchnis.

1. Ed. Schmidt, Die Winkel der kristallographischen Achsen der Plagioklase. Chemio der Erde, 1, $1915,358$.

2. G. Melczer, Daten zur genauen Kenntnis des Albits. Z. X. 40, 1905, 571.

8. C. Dreyer u. V. Goldschmidt, Über Albit von Gronland. Meddelelser om Grönland. 34, 1907, 7.

4. E. A. Wülfing, Dio Hăufungsmethode. Sitz.-Ber. Heidelb. Akad. Abt. A, 1916, 11. Abh., S. 12.

5. F. Klockmann, Kristallographische Untersuchung des Mikroklins und des Albits. Zeitschr. D. Geol. Ges. 34, $1882,410$.

6. S. L. Penfield, Ūber die Spaltbarkeit und Teilungsflächen beim Oligoklas und Albit. Z. X. 23, 1804, 263.

7. C. Hintze, Handbuch der Mineralogie. II, $1897,1449$.

8. Hosenbusch-Wülfing, Mikroskopische Physiographie. I, 1. - B. Aufl., 1921, 5.

Die meisten in der Natur vorkommenden Albite besitzen einen geringen Anorthitgehalt, der etwa $5 \%$ betrăgt. Ganz reiner Albit ohne die geringste Spur Anorthit scheint äußerst selten zu sein. Da nun die Untersuchung von Ed. Schmidt (1) gezeigt hat, $\operatorname{da} B$ in der ganzen Plagioklasreıhe die Winkel $\alpha, \beta, \gamma$ der kristallographischen Achsen eine Funktion der Zusammensetzung sind und sich nachweisbar ändern, so werden auch die Achsen- 
lăngen der verschiedenen Albite kleine Abweichungen zeigen. Wenn man daher die Morphologie des Albits feststellen will, muß dies an chemisch wohl definiertem Material geschehen. Die Einfügung der bisher für Albit angegebenen Winkel der Pinakoide oder der kristallographischen Acbsen in die von Ed. Schmidt aufgestellten Kurven ist nicht immer möglich, da Angaben über die chemische Zusammensetzung der betreffenden Albite fehlen. Auch schwanken die bisher für verschiedene Albite ermittelten kristallographischen Konstanten $(2,3,4)$ viel stärker, als sich aus der aller Wahrscheinlichkeit nach nur geringen Verschiedenheit der Zusammensetzung erklären ließe. Diese Schwankungen sind teils auf die unvollkommene Ausbildung der Albite, teils auf die Art der Messungen zurückzuführen; denn trotz der Häufigkeit dieses Minerals sind gut gewachsene Kristalle sehr selten und lassen auch dann noch in manchen Zonen gewisse Störungen erkennen, von denen man sich nur durch die Fülle der Beobachtungen und durch die Häufungsmethode (4) frei machen kann.

Von dieser Überlegung ausgehend, wurde mir von meinem verehrten Lehrer, Herrn Geh. Rat Wülfing, ein für Albit geradezu ausgezeichnet kristallisiertes Schweizer Vorkommen von der Alp Rischuna bei Vals in Graubünden übergeben, das ich im Mineralogisch-petrographischen Institut der Universität Ileidelberg unter Leitung von Herrn Geh. Rat Wülfing untersuchte.

\section{Identifizierung des Materials.}

Die zwölf vorliegenden Stufen bestehen mit Ausnahme von einer einzigen (VI), die eine größere Schieferunterlage besitzt, durchweg aus Albit und tragen z. T. auf beiden Seiten wohl ausgebildete Kristalle. Manche Stufen sind in der Ausbildung der Kristalle einander völlig gleich, so daß man sie von vornherein als idenlisch ansprechen möchte. Da aber bei ein und demselben Vorkommen die Plagioklase doch zuweilen etwas verschieden zusammengesetzt sein können, habe ich diese mehr qualitative Identität auch (quantitativ durch Bestimmung des spezifischen Gewichts, der Auslöschungsschiefe auf $P$ und $M$ und des Spaltungswinkels genau verfolgt. Hierzu wählte ich aus der Fülle des Materials vier bzw. fünf Stufen mit besonders schön ausgebildeten Kristallen, die die Nummern I, III, VI, IX, XI tragen.

Spezifisches Gewicht. Von den vier Stufen I, III, VI und XI wurden mit Hilfe von Lupe und Mikroskop je zehn 1-2 cmm große Stückchen von ganz reinem spaltenfreien Material ausgesucht und an.ihnen nach der Schwebemethode mit Thouletscher Lösung das spezifische Gewicht bestimmt. Alle Messungen wurden bei einer Zimmertemperatur von $18^{\circ}$ ausgeführt ${ }^{1}$. Die Mittelwerte für die einzelnen Stufen sind:

1) Zur Prüfung der Wostplialschen Wage sind noch gleichzeitig und bei derselben 'Temperutur von $18^{\circ}$ der Isländer' Doppelspat zu 2,715 und der Herkimer Quarz zu 2,652 richtig bestimmt worden. 


$$
\begin{array}{ll}
\text { Stufe I } & 2,623 \pm 0,003 \\
\text { Stufe III } & 2,624 \pm 0,002, \\
\text { Stufe VI } & 2,623 \pm 0,003, \\
\text { Stufe XI } & 2,623 \pm 0,003
\end{array}
$$

Das Mittel hieraus ist 2,623 bei $18^{\circ}$ mit einer Fehlergrenze, die man wobl nicht höher als $\pm 0,001$ anzusetzen braucht.

Auslöschungsschiefe auf $P$ und $M$. Zur weiteren Identifizierung des Materials wurde an einer Stufe (I) die Auslöschungsschiefe an je fünf Dünnschliffen nach $P$ und nach $M$ festgestellt und gefunden

$$
\Sigma_{P}=+3,5^{\circ} \pm 1^{\circ} ; \quad \Sigma_{M}=19,3^{\circ} \pm 1,5^{\circ} .
$$

Die Präparate nach $M$ waren wegen der unvollkommenen Spaltbarkeit nach dieser Fläche verhältnismäßig schwer herzustellen. Die große Fehlergrenze von $1 \frac{1}{2}^{\circ}$ ist aber mehr eine Folge der Unsicherheit in der Bewertung dieses oder jenes Spaltrisses,' was auch für die Bestimmung auf $P$ gilt. Über den Nachweis der Gleichartigkeit des Materials auf Grund des Spaltwinkels $P \bar{P}$ oder auch $P M$ s. S. 398 .

Chemische Zusammensetzung. Die chemische Zusammensetzung wurde an vorzüglich reinem Material der Stufe I von Fräulein Dr. Naima Sahlbom in Stockholm ausgeführt. Einem hierüber in den Sitzungsberichten der Heidelberger Akademie der Wissenschaften erscheinenden Aufsatz des Herrn Geh. Rat Wülfing entnehme ich die in der folgenden Tabelle verzeichneten Ergebnisse, die sich auf die Stellung des Rischunaer Albits in der Reihe der Plagioklase beziehen. Unter I sind die Gewichtsprozente der Analyse verzeichnet, unter II ist Eisenoxyd und Tonerde, Magnesia und Kalk, Kali und Natron vereinigt und Wasser nicht berücksichtigt. Unter III findet sich die Umrechnung auf $100 \%$ und unter IV die nach den einzelnen Grundbestandteilen berechneten Molekularprozente Anorthit, deren Mittelwert $3 \frac{1}{2} \% \pm 1 \frac{1}{2} \%$ beträgt. Mit dieser stimmt das spezifische Gewicht und die optische Untersuchung überein.

Chemische Zusammensetzung des Albits von Rischuna.

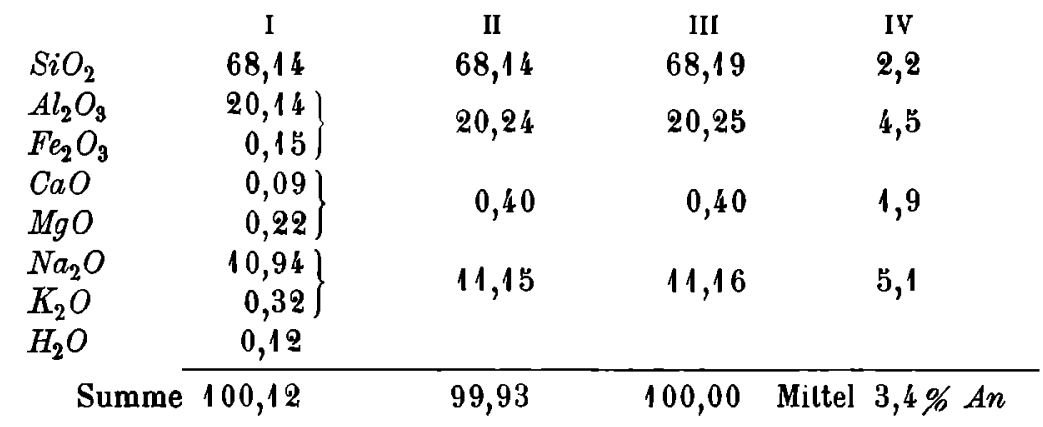




\section{Kristalltypen und Flächenbeschaffenheit.}

Einfache Kristalle finden sich auf keiner der vorliegenden Stufen; alle Kristalle sind vielmehr nach dem Albitgesetz verzwillingt, wozu bei vielen Stufen noch das Karlsbader Gesetz hinzutritt. Und zwar glaube ich wahrgenommen zu haben, daß die Albitverzwillingung alleine sich nur an kleinen Kristallen etwa bis zu einer Größe von $\frac{1}{2} \mathrm{~cm}$ und bei allen nach der Zone $M x$ langgestreckten Formen zeigt, während bei den größeren und ganz großen bis $3 \mathrm{~cm}$ messenden Kristallen und an allen nach $M$ stark ausgebildelen Formen noch die Karlsbader Verwachsung häufig auftritt. Andere Zwillingsgesetze wurden nicht beobachtet und vor allem solche nach dem Periklingeselz vergeblich gesucht.

Die Stufen tragen durchweg aufsitzende Kristalle, bei denen am oberen Ende die Zonen $P n$ und $o x$ und an der Unterseite die Zone $r M$ vorherrschen. Bei den nach dem Albitgesetz verzwillingten Kristallen sind meist zwei Individuen (Hauptindividuen) im Gleichgewicht ausgebildet, während zwischen ihnen öfters noch einige feine Lamellen auftreten, wie Fig. 1 zeigt. Mehrere gleich große Lamellen kommen nie zur Ausbildung. Orientiert man diese Albilzwillinge so vor dem Beschauer, da $B$ das eine Hauptindividuum in richtiger kristallographischer Stellung auf der linken Seile liegt wie in Fig. 1, 2 und 3, so zeigt dieses Individuum am oberen Ende der $c$-Achse häufig folgende Flächen:

$$
P(001), \quad x(\bar{\top} 01), \quad n(0 \overline{2} 1), \quad \delta(\bar{T} \bar{T} 2), \quad o(\bar{\top} T 1),
$$

und am unteren Ende

$$
\bar{y}(20 \bar{T}), \quad \bar{x}(10 \bar{T}), \quad \bar{r}(40 \overline{3}) \text {, und manchmal } \bar{p}(11 \bar{T}) \text {. }
$$

$e(021)$ wurde nicht und $\gamma\left(T_{12}\right)$ nur vereinzelt beobachtet. Bei den Prismen geht die Verwachsungsebene im allgemeinen durch $l(110)$ des Individuums in Grundstellung, wie bei Fig. 1, 2 und 3 zu sehen. Die Fläche $f(130)$ ist deshalb wie $\gamma$ nur selten vorhanden. Gemessen wurde $f$ nur ein einziges Mal.

Die Karlsbader $Z$ willinge treten bei den nach der $c$-Achse etwas gestreckten und wie schon bemerkt stark nach $M$ entwickelten Kristallen auf. Einfache Karlsbader $\mathrm{Z}$ willinge wurden nicht beobachtet und auch Vierlinge, bei denen wie in Fig. 7 zwei Albitzwillinge I, II und III, IV in Kallsbader Stellung getreten wären, konnten nicht gefunden werden. Von den hier eigentlich zu erwartenden vier Individuen, von denen also I und II, sowie III und IV je in Albitstellung stehen, fehlt bei einem linken Karlsbader $Z$ willing das Außenindividuum IV und bei einem rechten, durch den punktierten Teil in Fig. 7 angegebenen Karlsbader Zwilling das Individuum III'. Die Zone $n P \bar{P} \bar{n}$ (Fig. 2 und 4) fällt ungefăhr zusammen mit der Zone $\bar{x} \bar{p}$ des Individuums III oder mit $x p$ des Individuums IV' (s. Fig. 6 und 7, wo in Fig. 7 die Flächen $p$ und $\underline{\bar{p}}$ nicht gezeichnet sind). Sehr häufig ist bei 
einem linken Karlsbader $Z$ willing das Individuum II, bei einem rechten das Individuum I so schwach entwickelt, daß die Individuen I und III bzw. IV' und II alleine auftreten. Bei einer derartigen Flächenentwicklung (Fig. 6) kann man zunächst an einen einfachen Albitzwilling erinnert werden, wobei man $\bar{p}$ für $\underline{\bar{n}}$ bzw. $p$ für $n$ hält. Die Spaltflächen nach $P$ klären aber bald auf.
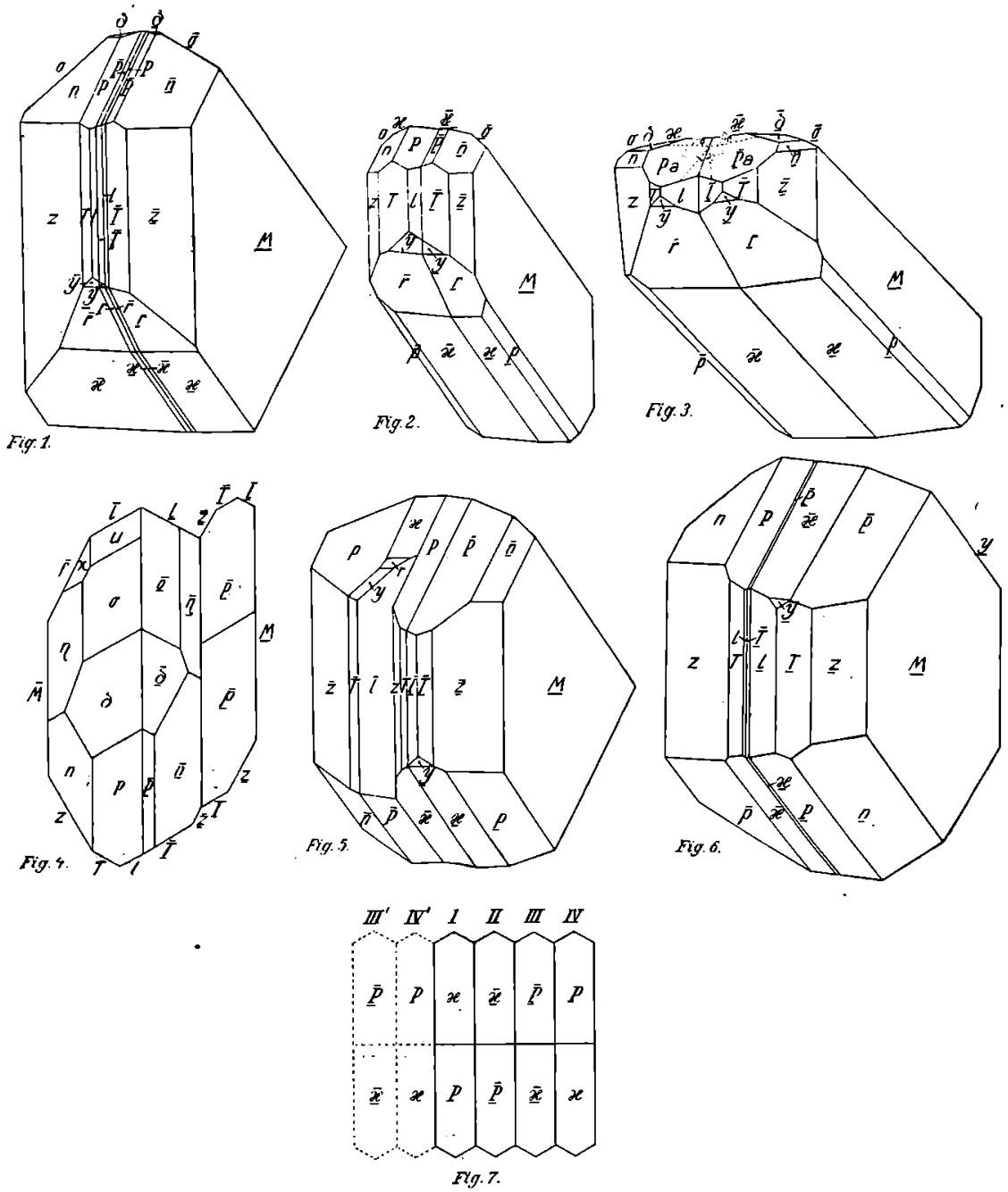

Die Stufen I und III zeigen ähnliche Entwicklung, nur sind bei der Stufe I die Kristalle häufig von Chlorit bestäubt und erfüllt, zeigen aber daneben auch sehr klare Individuen. Sie tragen auf der einen Seite die oben beschriebenen Karlsbader $Z$ willinge von $2-3 \mathrm{~cm}$ Ausbildung in tafelförmiger Entwicklung nach $M$, auf der andern Seite bis $1 \mathrm{~cm}$ große Kri- 
stalle, die entweder den großen Karlsbeder Kristallen in ihrer Ausbildung völlig gleichen oder nach der Zone $M x$ langgestreckte, einfacbe Albitzwillinge darstellen. Unter ihnen stecken viele kleine, besonders klare:Kriställchen, von denen solche von $2-5 \mathrm{~mm}$ Ausdehnung für genauere .Messung geeignet waren. Sie gleichen in ihrer Ausbildung den Figuren 2 und 3, seltener 1 und sind manchmal auch nach $M P$ in die. Länge gezogen.

Die großen Karlsbader Kristalle der Stufe III sind im Verhältnis zu ihrer Größe sehr gut ausgebildet. Das Innere der Kristalle ist etwas trübe. Nach dem oberen Teil hin treten die Verunreinigungen mehr zurück, so daß sich meist völlig wasserhelle Endteile der Kristalle mit glänzenden Flächen finden. An dem einen Individuum sind $n, P$, oft auch. $\delta$, am andern Individuum $\bar{p}$ und $\bar{y}$ glänzend und klar entwickelt, während die nach. hinten oben liegeñden Terminalfächen, wie z. B. $o, x, \bar{P}, \bar{\gamma}$ meist unvollkommen und öfters lückenhaft gewachsen sind.

Wie das Kopfbild (Fig. 4) eines durch Chlorit gefärbten Karlsbader Zwillings von guter Ausbildung zeigt, konnten (bei den Stufen I und III) außer den schon genannten Flächen die seltener vorkommenden Flächen $\eta(\overline{4} \overline{3} \mid), u(\overline{2} \overline{2} I)$ und $\chi(\overline{2} \overline{4} 1)$ beobachlet werden. Dazu kommt bei andern Kristallen noch das in Fig. 4 nicht abgebildete $\lambda(\overline{3} \overline{3} 2)$. $u$ wurde nur an dem einen hier gezeichnelen Kristall Nr. 168 wahrgenommen. $\chi$ wurde dreimal beobachtel, zweimal an großen Karlsbader Kristallen als kleine dreieckige Fläche mit Kanten gegen $l$ und $\eta$, einmal an einem kleinen Albitzwilling Nr. 97 von Stufe I, der zweikreisig durchgemessen wurde und der am hinteren Ende auch noch $\eta$ und $\lambda$ trug. $\lambda$ wurde außerdem auch noch an Kristall Nr. 117 von Stufe III gefunden, ebenfalls als schmale matte Fläche. Die Fläche $\psi$ ist glänzend, $\lambda$ und $u$ sind matt, - ließen sich aber trotz ihrer Kleinheit und ungenügenden Ausbildung sowohl durch Winkelmessung als auch durch den Zonenverband in ihren Indizes feststellen. $\eta$ ist an vielen Kristallen dieser. Stufen I und III, vor allem an den Karlsbader $Z$ willingen, neben $o$ bald schmal, bald breiler vorhanden, aber stets malt und bewachsen. Das Vorhandensein von. $\eta$ hängt mit dem Vorherrschen eines breiten $z$ und $n$ zusammen, eine Entwicklung, die bei den großen Karlsbader Kristallen häufig zu beobachten ist.

Stufe VI trägt auf einem Schieferbrocken aufsitzend fast nur Karlsbader Zwillinge von der gleichen Aushildung wie bei Stufe I und III, jedoch dünntafelig und von geringerer Ausdehnung. Zur Kristallmessung selbst sind die schmalen, bis $1 \frac{1}{2} \mathrm{~cm}$ nach $c$ langgestreckten Kristalle nicht geeignet. Dagegen läßt die Klarheit der Kristalle auf reines Material schließen, das eine sichere Bestimmung der Dichte vorzunehmen eriaubte. Auch konnten die Spaltwinkel sicher gemessen werden.

Die Stufe IX zeigt nur auf einer Seite gut ausgebildete Kristalle, die in ihrem Aussehen denen der chloritfreien, sebr reich entwickelten Seite 
von Stufe I gleichen. Die kleinsten Kriställchen zeigen wie Fig. 1 die Fläche $n$ sehr schön entwickelt. $n$ und $\bar{n}$ sind vorherrschend gegenüber dem sehr schmalen $P$ und $\underline{\underline{P}}$ und geben stets einheitliche Reflexe. Aus diesem Grund und da $n$ bei den übrigen Stufen oft Wachstumsstörungen zeigt, wurden die Kristalle dieser Stufe für die Messung des Winkels $n P$ ausgewählt (s. S. 402). Eigentümlich ist, daß bei dieser Stufe IX die beiden Individuen des Albitzwillings manchmal schief aneinander gewachsen sind, so daß die Zone $n P \underline{P} \underline{n}$ gestört war, was bei den andern Stufen nur vereinzelt beobachtet werden konnte und nur dann, wenn viele Zwillingslamellen zwischen den Hauptindividuen eingelagert sind.

Stufe XI ist allseitig mit einer Chloritschicht überzogen, aus der aber auf einer Seite wasserklare, $1-\ddot{3} \mathrm{~mm}$ große Kriställchen herausragen, deren Typus in Fig. 3 wiedergegeben ist. Ihr aufgewachsenes Ende ist meist von Chlorit durchtränkt. Auch sind die groß entwickelten Querdomen $x$ und $r$, nach denen die Kriställchen in die Länge gezogen sind, durch feinen Chloritstaub matt entwickelt. Dagegen ist das vordere Ende mit $P, M, z, T, l, \bar{y}$, $n$ und $\delta$ völlig wasserhell und besonders scharf ausgebildet. Genau die gleichen Formen fanden sich in einem chloritdurchtränkten Nest auf Stufe III. Die Unterseite wird hier stets von dem ganz bewachsenen Querdoma $\bar{r}$ eingenommen, das manchmal seitlich. von der schmalen, aber glänzend und klar ausgebildeten Fläche $\bar{p}$ begrenzt wird. Bei den aus dem trüben, grünlich gefärbten Untergrund herausgewachsenen Kristallen gewinnt man den Eindruck, als wenn die Verunreinigungen, die das Wachstum gestört haben würden, alle in dem Chlorit zurückgeblieben seien und dadurch das vordere Ende um so reiner zur Kristallisation gebracht hätten.

Die übrigen sieben Stufen des vorliegenden Albitmaterials kommen, wie gesagt, in ihrer Ausbildung den eben beschriebenen im großen und ganzen gleich.

Ein Überblick über die Stufen ergibt, daß folgende Flächen am Albit von Rischuna sicher nachgewiesen sind:

Bei allen Kristallen treten die Flächen $l, T, z, M, P, n, \delta, o, x, y, r$ an den Individuen in normaler und $Z$ willingsstellung auf. Nicht immer vorhanden sind $\eta$ und $p$; zuweilen, hauptsächlich an den Karlsbader Kristallen, wurde $f$ und $\gamma$ beobachtet. Dazu kommen noch die selteneren Flächen $u$, $\chi, \lambda$. Im ganzen konnten 18 Flächen sicher nachgewiesen werden.

Außer diesen Flächen sind noch zwei zu erwähnen, die je nur einmal beobachtet wurden und die, sowohl wegen des ganz vereinzelten Auftretens als auch wegen ihrer unvollkommenen Ausbildung, als unsicher angesehen werden müssen. An Kristall $\mathrm{Nr} .112$ von Stufe III liegt in der Zone $n \delta$ eine nach der Kante $n \delta$ stark gestreifte Fläche. Trotzdem sie nur auf $\pm 1^{\circ}$ zu messen war, konnte sie doch nach den einkreisigen und zweikreisigen Messungsresultaten als ( $\bar{T} \overline{5} 4)$ festgelegt werden. Diese Fläche 
wurde bis jetzt noch nicht am Albit beobachtet und möge den Buchstaben $\Sigma$ tragen. Die zweite unsichere Fläche wurde an Kristall Nr. 3 (Stufe XI) beobachtet. Sie ist sehr schmal mit scharfen Grenzen gegen $M$ und $\approx$ und besteht aus zwei um $0^{\circ} 49^{\prime}$ auseinander liegenden Teilflächen. Der Winkel gegen $(1 \bar{T} 0)$ beträgt $30^{\circ} 38^{\prime} \pm 25^{\prime}$ und führt zu der Fläche $\mathfrak{v}(1 \overline{4} 0)$, die von F. Klockmann (5) an Hirschberger Albiten beobachtet wurde. Auch in der Arbeit von C. Dreyer und V. Goldschmidt (3) ist die von Klockmann erwähnte Fläche (1 $\overline{4} 0)$ unter den unsicheren Flächen aufgeführt.

Die Ausbildung der Flächen ist meistens so typisch, da $B$ man an ihr die Flächen gut erkennen kann. $y$ ist stets glänzend und in der Regel einheitlich gewachsen; es liefert unter den gewachsenen Flächen die besten Reflexe. Auch $n$ ist stets glänzend, doch nicht von der gleichen Güte wie $y$, da die Fläche oft geätzt und in vizinale Flächen zerlegt ist mit symmetrischen Wachstumserscheinungen auf beiden Zwillingsflächen. Ähnlich' sind die gewachsenen $P$-Flächen, die stets aus zwei oder drei Teilfächen von hohem Glanz bestehen mit je nur einem hellen Reflex. Auch schmale Flächen zeigen diesen vizinalen Bau. In Fig. 3 ist diese Felderteilung der Flächen $P$ und $\underline{P}$ angedeutet und mit den Indizes $a, b, c$ bezeichnet. Die Abweichung $P_{a}: P_{b}$ beträgt in der $n P^{\prime}$-Zone $50^{\prime} \pm 10^{\prime}$; $P_{a}$ und $P_{c}$ decken sich ungefähr in dieser Zone. $P_{b}$ steht von $P_{c}$ in der Zone $P_{b} P_{c}$ über $1^{\circ}$ ab. Die Winkel sind einspringende. Keine der drei Vizinalflächen von $P$ fällt mit der richtigen Lage von $P$, wie sie aus der Spaltfläche erhalten wird, zusammen. Solche Abweichungen scheinen auch bei den Querdomenflächen $r$ und $x$ vorzukommen, da diese nie genau in der Zone $y$ Spalt- $P$ liegen. $r$ ist, wie schon erwähnt, stets matt und bewachsen. $x$ ist immer gekrümmt, fazettiert und geätzt und liefert stets mehrere undeutliche Reflexe. $\delta$ ist immer sehr klar und glänzend, aber selten einheitlich gewachsen. Oftmals sitzen kleine Wachstumshügel auf der Fläche $\delta$. $\mathrm{Zu}$ exakten Messungen sind Gewachsen- $P, r, x$ und $\delta$ nicht zu verwenden.

Die immer am oberen Ende des Kristalls vorhandene Grundpyramide $o(\bar{T} T 1)$ ist meist bewachsen und matt, manchmal jedoch, im Gegensatz zu $x$, von gutem Aussehen. Die gemessenen Winkel von $o$ gegen Spalt- $P$ und $n$ stimmen mit den berechnelen Werten gut überein. $p(\bar{T} 11)$ ist immer gut gewachsen, sowohl bei der vorherrschenden Entwicklung an den Karlsbader Zwillingen, als auch bei den schmalen, seitlich an $r$ anliegenden glänzenden Flächen an der Unterseite der nach $x M$ langgestreckten Formen (Fig. 2 und 3).

\section{Spaltbarkeit.}

Die Spaltung nach $P$ ist vollkommen und wird durch Einlagerung von Chlorit und Verunreinigungen nicht beeinflußt. Diese sebr gute Spaltbar- 
keit nach $P$ wurde zur indirekten Bestimmung des Spaltwinkels $P M$ aus dem Winkel $P \underline{P}$ benutzt. Auch bei allen andern fundamentalen Messungen ist stets der Winkel eines angespaltenen $P$ gegen die betreffende Fläche verwendet worden.

Die Spaltbarkeit nach $M$ konnte im allgemeinen nur als recht unvollkommen festgestellt werden. Gut war sie nur an der $Z_{\text {willingsgrenze der }}$ Albitzwillinge, besonders bei Einlagerung zahlreicher Lamellen nach dem Albitgesetz zwischen den beiden Heuptindividuen, wie dies auch schon S. L. Penfield beobachtete (6) und in C. Hintzes Handbuch vermerkt ist (7). Bei den großen Karlsbader Zwillingen ist die Spaltbarkeit nach $l(110)$ weit besser ausgebildet als die nach der Längsfläche. Bei den kleinen Kriställchen ist sowohl die Spaltbarkeit nach $M$ wie die nach $l$ sehr unvollkommen. Auf die Winkelmessungen von dem angespaltenen $P$ und $M$ gegen die angespaltene $l$-Fläche ist weiter unten S. 397 näher eingegangen.

Nach dem Vorschlag von Herrn Geh. Rat Wülfing wurde für die Herstellung der Spaltstückchen nicht Hammer und Meißel, sondern eine Zange benutzt (8), die man in der Richtung der gewünschten Spaltfläche angreifen ließ, ein Verfahren, bei dem sich die Spaltstückchen mit großer Sicherheit und Eleganz gewinnen lassen.

An den auf diese Weise hergestellten Spaltstückchen nach $P$ und $l$ wurde auch, ebenfalls bei größeren Kristallen, eine ziemlich unvollkommene Spaltung nach $o(T T 1)$ wahrgenommen, die Penfield (6) durch Pressen zwischen Bleiplatten im Schraubstock erhalten hat und als Teilbarkeit anspricht.

\section{Prismenzone.}

Wie aus der Beschreibung der Kristalle ersichtlich ist, sind auch bei den kleinsten ausgesuchten Kriställchen die meisten Flachen bewachsen und matt oder gestreift und angeătzt. Fast immer klar und glänzend sind unter den häufigen Flächen eigentlich nur $y(\overline{2} 01), l(110)$ und $T(1 \overline{1} 0)$ und allenfalls noch $n(0 \overline{2})$. Die in der Prismenzone neben $T$ und $l$ ausgebil-. dete Fläche $x(\mid \overline{3} 0)$ - ist nur bei schmalen Flächen einheitlich gewachsen. Bei den kleinen Kriställchen der Stufe XI sind $T$ und $l$ vorherrschend. - Bei vielen Krislallen der Stufen I, III und IX ist die Fläche $z$ breit entwickelt. Der einheitliche Teil dieser $x$-Fläche grenzt an $T$, während nach der andern Seite, also nach $M$, schmälere, um jeweils bis $1^{\circ}$ voneinander abweichende, vizinale Flächen auftreten. Bei den Karlsbader Kristallen hat $z$ stets die eben beschriebene Ausbildung. $T$ und $l$ sind hier meist schmal und oft sogar bei großen Kristallen noch einheitlich und glänzend. Die Längsfläche dagegen ist bei großen Kristallen immer gestreift und sogar bei den kleinen Kriställchen nur selten gut ausgebildet. Sie liefert. 
stets mehrere, bis $1^{\circ}$ auseinander liegende Reflexe, die innerhalb der Prismenzone abgelenkt sind. Das gespaltene $M$, sobald dies trotz der schlechten Spaltbarkeit gelegentlich als gute Fläche erhalten wird, erscheint stets in richtiger Lage. An $T$ wurde vizinaler Bau nur selten beobachtet, an allen gemessenen Kriställchen nur dreimal. In allen andern Fällen konnte an $l$ und $T$, auch wenn die Zwillingsnaht hindurchging, völlig einheitlicher Bau wahrgenommen werden.

Bei der sehr guten Beschaffenheit dieser beiden Flächen $l$ und $T$ schien es naheliegend, sie für die Berechnung des Achsenverhältnisses zu benützen. Die vorgenommenen Messungen, die mit ihren Werten in den nachfolgenden Tabellen enthalten sind, zeigen jedoch, daß nicht nur $M$ sondern sämtliche Prismenflächen innerhalb der Zone abgelenkt sind, trotz der eben erwähnten guten Flächenbeschaffenheit. Diese Stürungen werden außerdem erwiesen durch die $\mathrm{Ablenkung}$ der Prismen aus den Terminalzonen $l P \delta o \bar{l}$ und $T P \gamma p \bar{T}$. Auf solche Störungen baben bereits C. Dreyer und V. Goldschmidt (3) bei den grönländischen Albiten bei selbst noch so guten Rellexen hingewiesen.

Da wir es immer mit Albitzwillingen zu tun baben, erlauben die Kristalle
1. die direkte Messung $T z, T l$,
2. die indirekte Messung $T M, l M, \approx M$

aus den Winkeln $T \underline{T}, \underline{l} \overline{\underline{l}}, \boldsymbol{x}_{\bar{z}}$.

Die ganze Unruhe der Flächenlagen in der Prismenzone geht aus den Häufungsbildern Fig. 8-12 deutlich hervor. Von einer ausgesprochenen Häufung kann ja wohl nirgends die Rede sein. Versucht man aber trolz der Zerstreuung Millelwerte zu bilden, so gelangt man, wenn man nur die durch die Bögen in den Fig. 8-12 angedeutete Auswahl trift, zu folgenden Zahlen:

$$
\begin{aligned}
& \text { aus Fig. } 9 \text { Tl } \quad=59^{\circ} 10^{\prime} \pm 14^{\prime} \text {, } \\
& \text { > } 11 l i \underline{1}=5819 \pm 16 \text {, } \\
& \text { ॰ } 10 T \bar{T} \quad=5956 \pm 7 \text {, } \\
& \Rightarrow \quad 12 l \underline{T}=\underline{i} T=046 \pm 29 \text {, } \\
& \triangle 8 z T=2937 \pm 7 \text {. }
\end{aligned}
$$

Hier kontrollieren sich

$$
\begin{aligned}
& T l+l \bar{T}=T \underline{\bar{T}}=59^{\circ} 56^{\prime}, \\
& T l-\underline{\bar{l}} T=l \underline{\underline{\underline{l}}}=58 \quad 2 k .
\end{aligned}
$$

Aus dem Zonenverband ergibt sich nach dem Sinusdoppelverhältnis der Wert für $T \approx$ zu $29^{\circ} 44^{\prime}$, der von dem gemessenen Wert $T z=29^{\circ} 37^{\prime}$ um etwas weniger, aber immer noch um $7^{\prime}$ abweicht. Diese ziemlich gute Übereinstimmung ist aber doch kein genügender Beweis für die richtigen Lagen der Flächen, denn diese passen nicht gut auf den Zonenverband der Terminalबächen $l P \delta_{o} \bar{l}$ und $T P \gamma p \bar{T}$. 
Man erhält aus allen diesen zahlreichen Beobachlungen die Vorstellung, daß die Flächen $l, T, z$ einer bestimmten Mitlellage zuneigen, die aber nicht der richtigen Lage am rational aufgebauten Kristall entspricht, sondern aus irgend einem uns unbekannten Grund eine Störung erfahren hat. Die Berechnung des Achsenverhältnisses mußte deshalb ohne Verwendung der Prismenwinkel vorgenommen werden. Welcher Weg auf Veranlassung

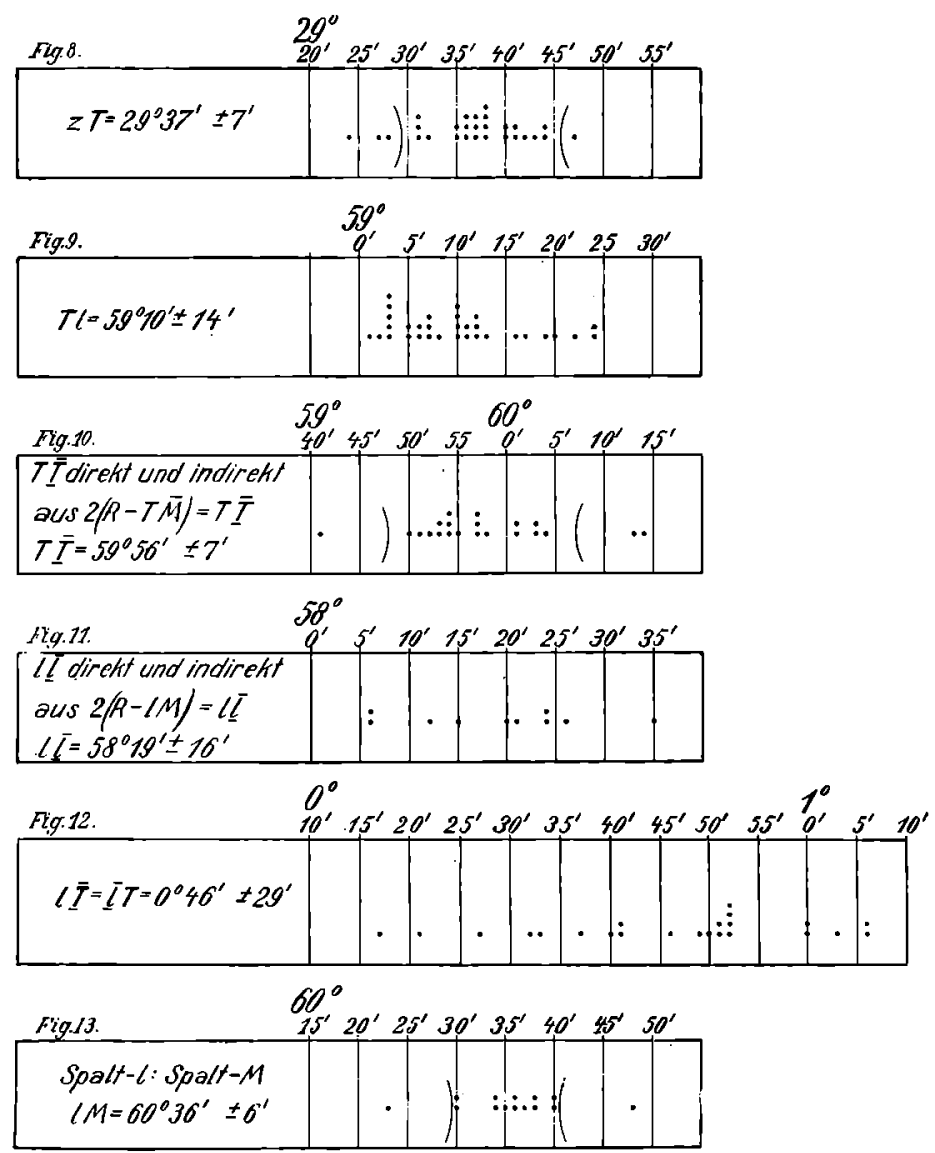

meines verehrten Lehrers hierzu eingescblagen wurde, wird Seite 397 auseinandergesetzt werden, hier sei nur vorgreifend das bemerkenswerte Ergebnis mitgeleilt, daß bei einer Rückwärtsberechnung der Winkel aus dem endgültigen Achsenverhältnis die Fläche $T$ ziemlich ungestört und die Fläche $l$ sebr stark abgelenkt erscheint. Die nachfolgende Gegenüberstellung der gemessenen und berechneten Winkel der Flächen $l$ und $T$ gegeneinander und gegen $M$ und $P$ möge dies illustrieren. 


$\begin{array}{cc}\text { Gemessenc Werte } & \text { Berechnete Werte } \\ T \bar{M}=60^{\circ} 2^{\prime} \pm 7^{\prime} & 60^{\circ} 3,2^{\prime} \\ T P==6910 \pm 4 & 69 \quad 10,4 \\ M l=6050 \pm 8 & 6037,3 \\ P l=6: 7 \quad 7 & 65 \quad 8,5 \\ T l=5910 \pm 14 & 5919,5 .\end{array}$

Wie schon S. 394 erwähnt, fiel auf, daß die in Hintzes Handbuch der Mineralogie nur als unvollkommen bezeichnete Spaltbarkeit nach $l$ an dem Rischuna-Albit an mehreren Stufen gut und bedeutend besser ausgebildet ist als die nach der Längsfläche. Diese vor allem an den großen Karlsbader Kristallen der Stufen I und III hergestellte Spaltfäche $l$ erlaubt die Winkel $P l$ und $P M$ zu messen und führt zu Werten, die von den Winkeln der gewachsenen $l$-Fläche erheblich abweichen, aber mit jenen Winkeln gut übereinstimmen, die sich aus dem schließlich gefundenen Achsenkreuz ergeben. Der gemessene Wert für Spalt-l zu Spalt-M, wie er sich aus 1: Messungen der Stufen I und III unter Ausschluß von zwei Messungen ergibt (s. Häufungsbild Fig. 13), beträgt $60^{\circ} 36,0^{\prime} \pm 5^{\prime}$. Der aus dem aufgestellten Achsenkreuz berechnete Wert ist, wie schon oben gesagt, fast der gleiche, nämlich $60^{\circ} 37,3^{\prime}$, während das gewachsene $l$ zu dem Wert $60^{\circ} 30^{\prime} \pm 8^{\prime}$ führt. Die gespaltene $l$-Fläche liegt also an der richtigen Stelle, die gewachsene weicht etwa $13^{\prime}$ nach vorne davon ab und hat demnach die Tendenz, von $M$ wegzurücken.

\section{Gang der Untersuchung zur Ermittlung des Achsenkreuzes.}

Meine erste Absicht, die Winkel der Prismen $T$ und $l$ gegeneinander und gegen $y$ und das angespaltene $P$ als Fundamentalwinkel zu verwenden, war mit Erkenntnis der Ablenkung der Prismen hinfällig geworden. Es kamen nunmehr als Flächen für die Fundamentalmessung überhaupt nur in Betracht die Spaltflächen $P$ und $\underline{\underline{P}}$, dazu $y, \ddot{y}$ und $n, \bar{n}$. Von den übrigen nicht prismatischen Flächen wäre nur an $o$ und $p$ zu denken. Die Fläche $o(T \bar{T} 1)$ ist zwar, wie schon erwähnt, zuweilen sehr gut ausgebildet, öfters aber matt, bewachsen und gestreift, tritt aber doch zu selten auf, und auch $p$ ist nicht oft genug beobachtet worden, um nach der Häufungsmethode behandelt zu werden. - Da wir es immer mit Albitzwillingen zu tun haben, ist zu den Winkeln, die $y, P, n$ miteinander bilden, noch der Winkel der drei Flächen gegen $M$ gegeben. Diese vier Flächen genügen aber nicht zur Bestimmung des Achsenkreuzes, da $M, P$ und $n$ in einer Zone liegen.

Nach dem Vorschlag meines verehrten Lehrers habe ich als fünfte Fläche den Pol der Prismenzone hinzugenommen, d. h. den einkreisigen Messungen die zweikreisigen hinzugefügt. Dieses Verfahren war deshalb 
aussichtsreich, weil die Prismenzone zwar nicht in der Lage ihrer Flächen innerhalb der Zone, wohl aber als Zone völlig ungestört ist und sich daher zur Einstellung ihres Pols für die zweikreisige Messung gut eignet. Auf diese Weise erhält man außer den fünf Winkeln zwischen $M, P, y$ und $n$ untereinander noch vier weitere Winkel, so daß der Albit von Rischuna durch folgende neun Winkel in seinem Aufbau festgelegt ist (Fig. 28, S. 403):
1. $P M=(001)(010)$,
亏. $n P=(0 \overline{2} 1)(001)$,
2. $y M=(\overline{2} 01)(010)$,
6. $C P=[001](001)$
3. $y P=(\overline{2} 01)(001)$,
7. $C y=[001](\overline{2} 01)$,
4. $n y=(0 \overline{2} 1)(\overline{2} 01)$,
8. $C n=[001](0 \overline{2} 1)$
9. $C M=[001](010)=90^{\circ}$.

Mit dem zweikreisigen Goniometer wurden nur die Poldistanzen von $M, P, y$ und $n$ festgelegt und die Bestimmungen der Meridianwerte unterlassen, da diese mit um so größerer Ungenaüigkeit verbunden sind, je kleiner ihre Poldistanzen werden.

\section{Fundamentalwinkel.}

1. Der Spaltwinkel $P \underline{\bar{P}}$ und $P M$. Der sicherste aller gemessenen Winkel ist der Spaltwinkel $P \underline{\bar{P}}$. Aus seiner Konstanz geht die ungestörte Lage der Spaltflächen hervor. Da alle Kristalle Albitzwillinge sind, konnte der Winkel $P \underline{P}$ sehr leicht erhalten werden. Er diente deshalb auch als ausgezeichnetes Vergleichselement der verschiedenen Stufen untereinander und bildet weiterhin einen wichtigen Grundstein für die Fundamentalmessung. Er wurde hauptsächlich an vier Stufen (I, III, VI und XI) je an einer sehr großen Zahl ausgewählter Spaltstückchenvon $\frac{1}{2}-2 \mathrm{~mm}$ Dicke gemessen. An Stufe IX waren die Spaltwinkel $P \underline{\bar{P}}$ von mir nur vereinzelt gemessen worden, doch hat Herr Assistent Dr. J. Kratzert, dem ich auch an dieser Stelle meinen besten Dank dafür ausspreche, die Güte gehabt, diese Messungen nachzuholen.

Bei den Stufen I und III haben die großen, im Innern trüben Kristalle auf der einen Seite, und die sehr klaren kleinen Kristalle auf der andern Seite trotz der verschiedenen Ausbildung bis auf wenige Zehntel Minuten denselben Wert für die Spaltwinkel $P \bar{P}$ ergeben. Auch hat sich diese Konstanz bei der Messung der äußeren und inneren Teile der großen Kristalle gezeigt, womit nachgewiesen ist, daß diese trotz der Größe keinen zonaren Aufbau haben. Die Einheillichkeit des Materials jeder Stufe ist damit auch auf morphologischem Weg dargelan, wie dies schon durch das spezifische Gewicht und die Auslöschungsschiefe geschehen,

Nach dieser sorgfälligen Voruntersuchung kann das Material von Rischuna in seiner Gesamtheit als einheillich betrachtet werden. Aus dem Winkel $P \underline{P}$ läßt sich der Winkel $P M=R-\frac{1}{2} P \underset{P}{P}$ berechnen, dessen Fehler 
sich auf die Hälfte des an $P \underline{\underline{P}}$ gemessenen verringerl. Der Spaltwinkel $P M$ wurde auch direkt gemessen, jedoch sind diese Messungen weniger zahlreich und weniger zuverlässig, da das gespaltene $M$ nicht so gut erhalten werden kann. Trolzdem zeigt sich eine sehr schöne Übereinstimmung der

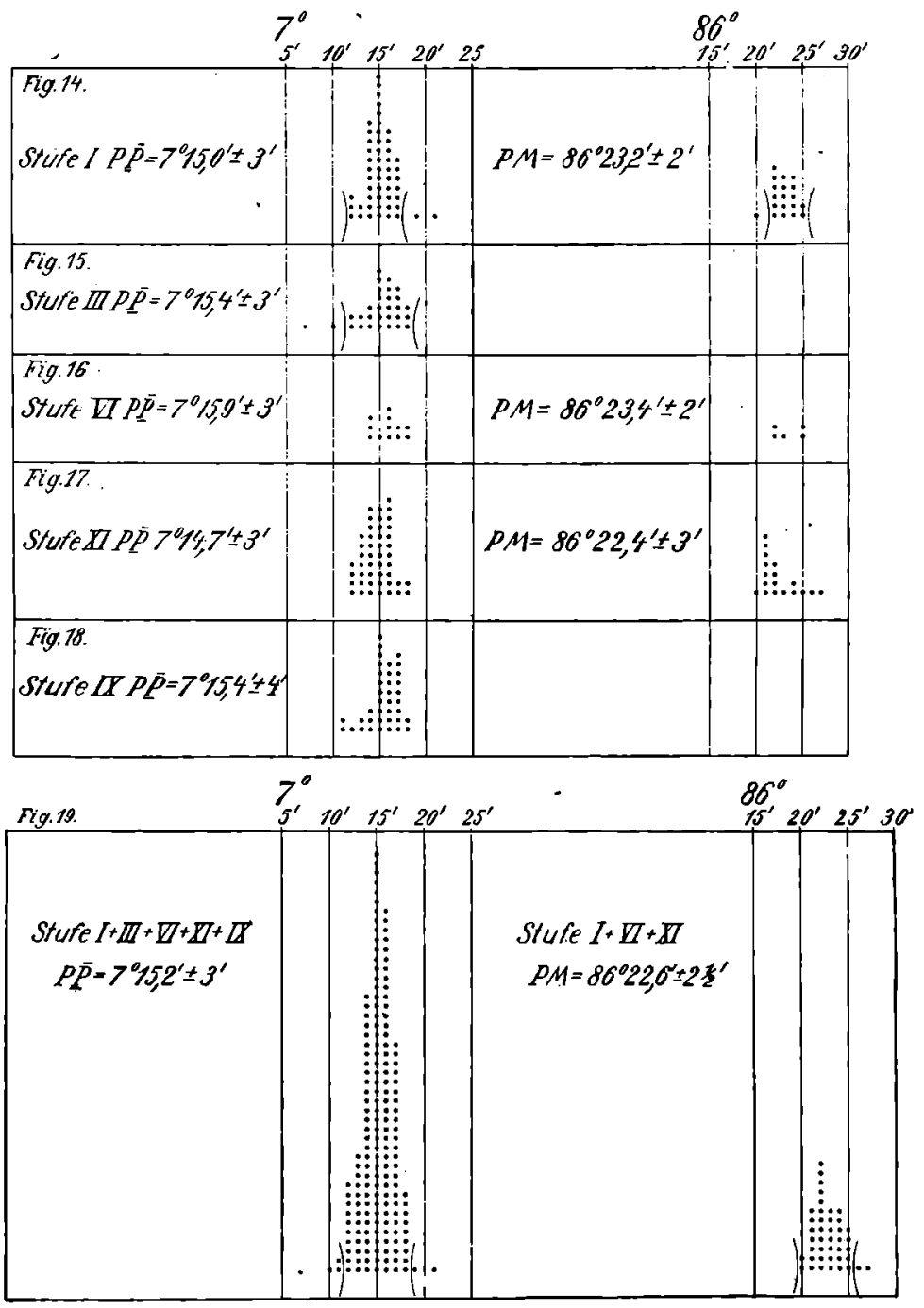

Werle. Am besten bekommt man den Überblick auch hier wieder durch die Häufungsbilder. In den Fig. 14-18 sind die Messungen an den einzelnen Stufen und in Fig. 19 an sämtlichen Stufen zu einem Bilde vereinigt. Es sind gemessen worden: 


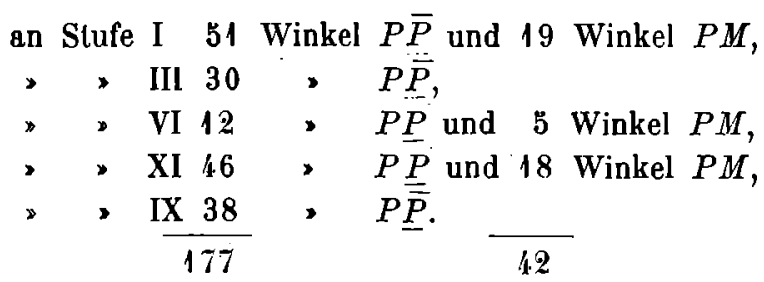

An Stufe VI wurde eine geringere Zahl von Messungen ausgeführt, weil aus ihnen die Identität mit den andern Stufen schon deutlich hervorging. Von diesen 177 Winkeln $P \dddot{P}$ liegen nach dem Gesamthäufungsbild in Fig. 19 nur sechs abseits. Nach ihrer Streichung beträgt der Winkel $P \underline{P}$ im Mittel $7^{\circ} 15,2^{\prime} \pm 3^{\prime}$, woraus sich der Spaltwinkel $P M$ zu $86^{\circ} 22,4^{\prime} \pm 11^{\prime}$ ergibt. Die direkte Messung $P M$ aus 40 von 42 Einzelmessungen führt in guter Übereinstimmung hiermit zu dem Mittelwert von $86^{\circ} 22,6^{\prime} \pm 21^{\prime}$. Im Mittel aus allen $171+10$ Messungen folgt für den Spaltwinkel des Albits von Rischuna

$$
P M=86^{\circ} 22,5^{\prime} \pm<21 .
$$

2. und 3. Die Winkel $y M$ und $y P$. Sehr zuverlässig sind auch die Winkel $y M$ und $y P$, da $y$ die bestgewachsene Fläche des Albits ist. Der Winkel $y M$ wird aus dem Zwillingswinkel $y \bar{y}$ erhalten, konnte aber nicht an allen Kristallen gemessen werden, da oft die Zwillingslläche $\bar{y}$ zu $y$ fehlt, wenn das eine Individuum des Albitzwillings vorherrschend ist. An Stufe XI wurde auch 4 mal $M y$ direkt gemessen gegen ein gespaltenes $M$, was im Häufungsbild Fig. 20 durch die umkreisten Punkte angedeutet ist. Man sieht in diesem Häufungsbild das Herausfallen von 3 Werten aus dem Haufen der übrigen 15 , welch letztere zu dem Mittelwert führen $y \bar{y}$ $=5^{\circ} 24,3^{\prime} \pm 4^{\prime}$ oder zu

$$
y M=87^{\prime} 17,8^{\prime}+2^{\prime} .
$$

$y P$ konnte an verschiedenen Kristallen $42 \mathrm{mal}$ bestimml werden, wie dies aus dem Häufungsbild Fig. 21 zu ersehen ist. Mit $\Lambda$ usschluß von zwei seitlichen Werten gelangt man hier zu dem Mittelwert

$$
\text { y } P=82^{\circ} 8,8^{\prime} \pm 5^{\prime} \text {. }
$$

4. und 5. Die Winkel $n y$ und $n P$. Die Flächen $n$ und $\bar{n}$, die an allen Kristallen vorkommen, zeigen, wie schon erwähnt, nicht die Güte der Spaltfläche $P$ und der gewachsenen Fläche $y$. Oft sind die $n$-Flächen so groß, da $\beta$ die gewachsenen $P$-Flächen dagegen zurücktreten. $n$ erscheint dann in Teilflächen zerlegt und oft wie geäzt und gestreift. Eine Störung der Lage gegen $y$ läßt sich nicht unmiltelbar erkennen, weil hier keine gute dritte Fläche der Zone berangezogen werden konnte. Anders bei der Zone $P n$, wovon alsbald die Rede sein wird: Das Häufungsbild Fig. 22 enthält 21 Messungen, die unter Ausschluß von zwei Winkeln zu dem Mitlelwert führen

$$
n y=86^{\circ} 12,3^{\prime} \pm 3^{\prime} \text {. }
$$


In der Zone $n P$ ist, wie gesagt, die weniger gute Entwicklung von $n$ insofern deullich, als sich bei einer größeren Zahl von Kristallen der Stufen III und IX ein Herausfallen der Flächen $n$ uud $\underline{\bar{n}}$ aus der Zone $P \underline{P}$ beobachten läßt, während bei vielen andern Kristallen keine -Zonenstörung wahrgenommen werden kann. Nur durch sehr zahlreiche Messungen läßt
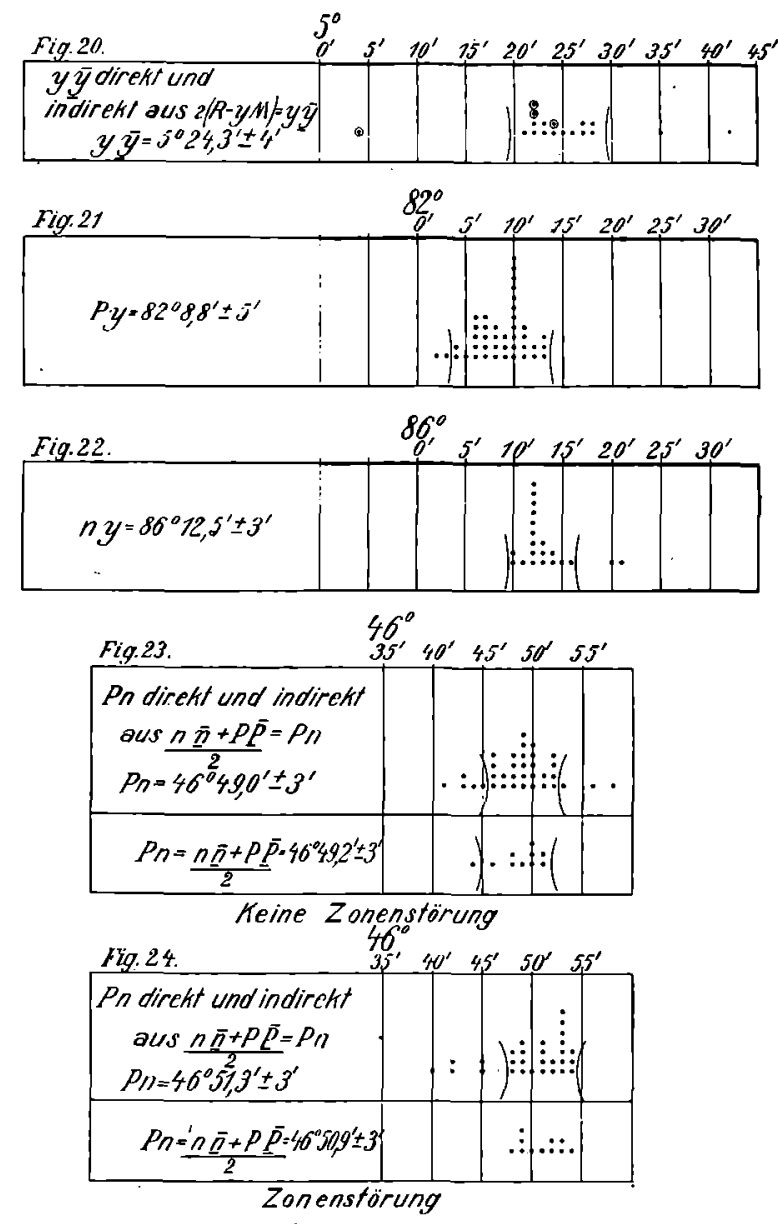

sich eine Übersicht gewinnen, die durch die Häufungsbilder in Fig. 23 und 24 wiedergegeben ist. Die Mittelwerte dieser Messungen zeigen nun, daß bei den Kristallen ohne sichtbare Zonenstörung die Flächen $n$ und $\underline{\bar{n}}$ viel besser auf die weiter unten mitgeteilten Ausgleichswerte passen, aber ihre wahre Lage doch noch nicht vollständig erreichen. Der für $n P$ erhaltene Mittelwert an Kristallen ohne Zonenstörung ist 


$$
\begin{array}{ccc}
\text { gemessen } & 46^{\circ} 49,0^{\prime} \pm 3^{\prime} & \text { berechnet }
\end{array}
$$

und an Kristallen mit Zonenstörung

$$
n P \quad 46^{\circ} 51,3^{\prime} \pm 3^{\prime} \quad 46^{\circ} 47,8^{\prime} \text {. }
$$

Erleichtert wurde auch hier wieder das Häufungsverfahren durch das Vorherrschen von Albitzwillingen, die die Messung von $n P$ in der Regel zweimal gestatteten. Auch war durch Messung von $n \bar{n}$ eine erwünschte Bestimmung des Winkels $n M$ möglich, der den Winkel $n P$ zu dem bekannten Winkel $P M$ ergänzt und damit kontrolliert. In dem unteren Teil der Fig. 23 und 24 sind die aus $n \underline{\bar{n}}$-Messungen und dem bekannten Wert $P M$ erhaltenen Winkel $n P$ gesondert dargestellt, in dem oberen Teil sind sie mit den direkten $n P$-Messungen vereinigt. Man sieht, da $\beta$ die beiden abhängigen Winkel $n M$ und $n P$ gut zusammenpassen. - Die Ablenkung von

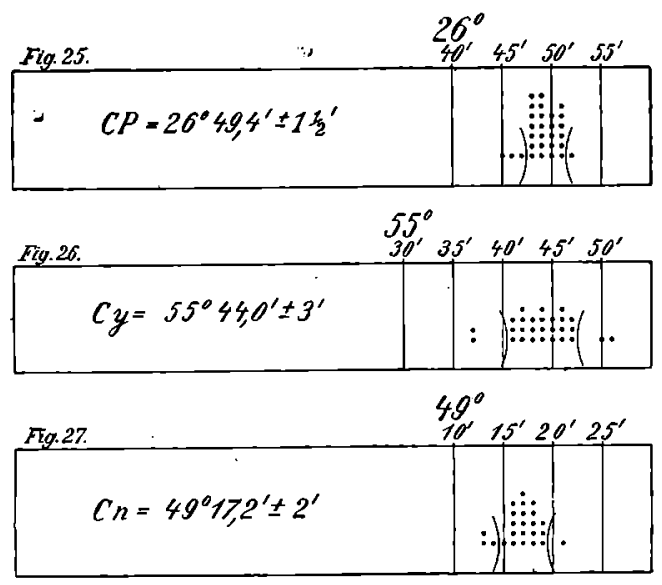

$n$ aus der Zone $P M$ im Sinne der Fig. 28 wurde auch durch Messung mit dem zweikreisigen Goniometer nachgewiesen.

6., 7., 8. und 9. Die Abstănde der Flächen $P, y, n$ und $M$ vom Pol $C$ der Prismenzone. Für die nach der Überlegung auf S. 397 erforderliche Bestimmung der Polabstände der Flächen $P, y, n$ und $M$ wurden 19 der bereits einkreisig gemessenen Kristalle auch der zweikreisigen. Messung unterworfen und in allen Einzelheiten, die unter dem Goniometer zugãnglich waren, beobachtet.

Das Häufungsbild Fig. $2 \ddot{3}$ zeigt 29 Werte von $C P$ an verschiedenen Stufen gemessen; der Mittelwert aus 23 von diesen 29 Messungen beträgt

$$
C P=26^{\circ} 49,4^{\prime} \pm 1 \frac{1}{2}^{\prime} \text {. }
$$

Er ist neben $P \underline{P}$ und $P M$ der sicherste von allen gemessenen Winkeln und zeigt nicht nur die uns schon bekannte Vortrefflichkeit der gespaltenen 
$P$-Fläche, sondern auch die Genauigkeit der Ausbildung der Kristalle nach der Prismenzone, die ja zum Einstellen der $C$-Fläche gedient hat.

Die Werte für $C y$ sind im Häufungsbild Fig. 26 zusammengestellt. Wie aus letzterem zu ersehen, muß die Fehlergrenze etwas größer angenommen werden als bei $C P$. Der Mittelwert aus 24 von 28 Messungen ist

$$
C y=55^{\circ} 44,0^{\prime} \pm 3^{\prime} \text {. }
$$

Cn konnte nur an 14 von den 19 Kristallen zusammen $24 \mathrm{mal}$ gemessen werden, da $n$ nicht immer genügende Schärfe besitzt. Die geringere Güte der Fläche $n$ ist auch hier, wie bei der Messung von $n P$ durch das Auftreten verschiedener Doppelreflexe, die glücklicherweise nur wenige Minuten voneinander abweichen, erwiesen. Die nahe beieinander liegenden Doppelreflexe sind in ihren Mittelwerten mit verwandt worden. Häufungsbild Fig. 27 zeigt die Verhältnisse und läßt erkennen, wie die

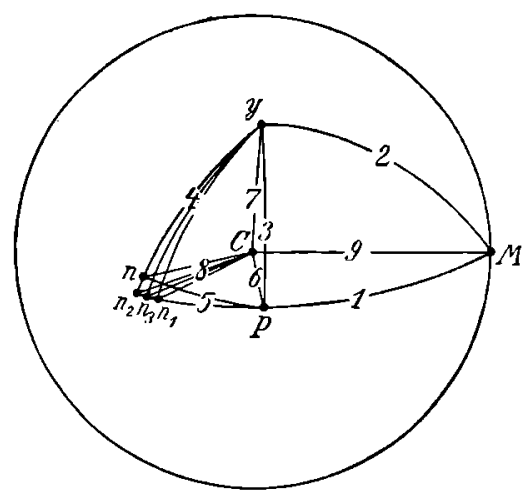

Fig: 28

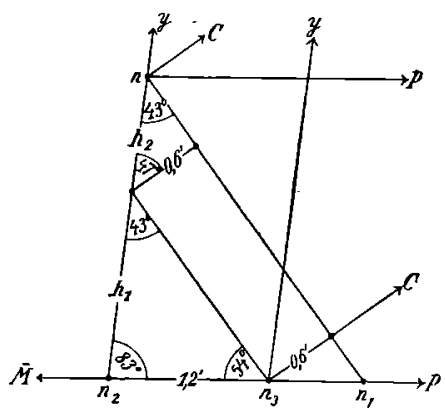

Fig. 29.

Häufungsmethode auch bei relativ weniger guten Flächen gute Ergebnisse liefern kann. Der Mittelwert aus 20 von 24 Messungen ist

$$
C n=49^{\circ} 17,2^{\prime} \pm 2^{\prime} \text {. }
$$

Als vierter Polabstand ergibt sich der Winkel $C M$ unmittelbar zu $90^{\circ}$.

\section{Ausgleich der Fundamentalwinkel und Berechnung des Achsen- kreuzes.}

Hiermit ist die Lage der vier Kristallflächen $P, M, y$ und $n$ zueinander und zum Pol der Prismenzone durch neun Winkel bestimmt, die hier noch einmal zur Übersicht zusammengestellt werden mögen.
1. $P M=86^{\circ} 22,3^{\prime} \pm 1 \frac{1}{2}$,
5. $n P=46^{\circ} / 49,0^{\prime} \pm 3^{\prime}$
2. $y M=8 \pi \quad 17,8 \pm 2$,
6. $C P=26 \quad 49,4 \pm 1 \frac{1}{2}^{\prime}$,
3. $y P=82 \quad 8,8 \pm: 3^{\prime}$,
7. $C y=80344,0 \pm 3^{\prime}$,
4. $n y=86 \quad 12,5 \pm 3^{\prime}$,
8. $C n=49 \quad 17,2 \pm 2^{\prime}$,
9. $C M=90^{\circ}$. 
Diese Winkel sind untereinander auszugleichen, was ich nach dem Vorschlag von Herrn Geh. Rat Wülfing folgendermaßen durchführte (s. Fig. 28 und 29).

Die sehr sicher bestimmten Winkel $P M$ und $C P$ wurden mit $C M\left(=90^{\circ}\right)$ unverändert in die weitere Rechnung übernommen. Alsdann wurde die Lage der Fläche $n$ korrigiert. $n$ sei die tatsächlich beobachtete Lage der Fläche $(0 \overline{2} 1)$, die den Mangel hat, nicht genau in die Zone $M P \bar{M}$ zu fallen und die nun durch kleine Korrekturen an $P n$ und $C n$ in die zonenrichtige Lage zu bringen ist.

$n_{1}$ sei eine zonenrichtige Lage von $(0 \overline{2} 1)$ mit dem gemessenen Winkel $C n_{1}(=C n)$ und dem nicht gemessenen Winkel $P n_{1}$.

$n_{2}$ sei eine zonenrichtige Lage von (0 $\left.\overline{2} 1\right)$ mit dem gemessenen Winkel $P n_{2}(=P n)$ und dem nicht gemessenen Winkel $C n_{2}$.

$n_{3}$ sei eine zonenrichtige Lage von (0的) nach Ausgleich der Winkel $C n$ und $P n$. Dieser Ausgleich geschah rechnerisch folgendermaßen.

1. $\triangle C M P$ (Fig. 28) bleibt starr und daher auch Winkel $C P \bar{M}\left(=C P n_{1}\right.$ $\left.=C P n_{2}=C P n_{3}\right)$.

2. $\triangle C P n_{1}$ führt mittelst $C P, \triangle C P n_{1}$ und $C n_{1}(=C n)$ zu den Werten $P n_{1}, \triangle P n_{1} C$ und $\triangle n_{1} C P$.

3. $\triangle C P n_{2}$ führt mittelst $C P, \triangle C P n_{2}$ und $P n_{2}(=P n)$ zu den Werten $P n_{2}, \triangle P n_{2} C$ und $\Varangle n_{2} C P$.

4. $\triangle C P n_{3}$ führt mittelst $\measuredangle C P n_{3}(=C P \bar{M}), \quad \triangle_{\frac{1}{2}}\left(n_{1} C P+n_{2} C P\right)$ und $\triangle \frac{1}{2}\left(P n_{1} C+P n_{2} C\right)$ zu den ausgeglichenen Werten $P n_{3}$ und $C n_{3}$. Man erhält

\begin{tabular}{c||c|c}
\hline \multicolumn{1}{|c||}{ Ausgleichsrechnung } & \multicolumn{3}{c}{ M essung } \\
& $\begin{array}{c}\text { an scheinbar ungestörten } \\
\text { Kristallen }\end{array}$ & $\begin{array}{c}\text { an deutlich gestörten } \\
\text { Kristallen }\end{array}$ \\
\hline \hline$P n_{3} 46^{\circ} 47,8^{\prime}$ & $P n=P n_{2} 46^{\circ} 49,0^{\prime} \pm 3^{\prime}$ & $46^{\circ}: 1,3^{\prime} \pm 3^{\prime}$ \\
$C n_{3} 49 \quad 17,8$ & $C n=C n_{1} 4917,2 \pm 8$ & -
\end{tabular}

Der Vergleich dieser Winkel zeigt, daß auch bei den scheinbar völlig ungestörten Kristallen Abweichungen von $n$ etwa senkrecht zu $C n$ eintreten, wie dies in Fig. 28 angedeutet ist.

Entsprechend der Verschiebung der Lage von $n$ nach $n_{3}$ muß nun zunächst auch der Winkel $n y$ korrigiert werden. Dazu entwirft man eine Zeichnung wie Fig. 29, die die Verhältnisse der stereographischen Projektion in der Gegend von $n$ stark vergrößert und entsprechend dem kleinen Kugelbereich in ebenen Dreiecken darştellt. Die Flächen $P, C$ und $y$ hat man sich hier weit draußen vorzustellen, so daß $n P\left\|n_{2} P, n_{2} y\right\| n_{3} y, n C \|$ $n_{1} C \| n_{3} C$. Es genügt, wenn die Winkel $P n y$ und $P n C$ auf ganze Grade abgerundet bekannt sind. Die Änderung der Strecke $n y$ in $n_{3} y$ beträgt $n_{3} y-n y=h_{1}+h_{2}$, also nach den ebenen Dreiecken der Fig. 29 


$$
h_{1}=1,2^{\prime} \frac{\sin 54^{\circ}}{\sin 43^{\circ}}=1,42^{\prime} ; \quad h_{2}=\frac{0,6^{\prime}}{\sin 43^{\circ}}=0,88^{\prime} .
$$

Hiernach ist $n_{3} y=n y+h_{1}+h_{2}=n y+2,3^{\prime}=86^{\circ} 14,8^{\prime}$.

Nachdem somit durch die starre Verwendung von $C M, M P$ und $P C$, sowie durch den Ausgleich von $P n$ und $C n$ die Fläche $n$ nach $n_{3}$ verlegt ist, nachdem ferner der Winkel $n y$ hiermit in Übereinstimmung gebracht ist, müssen noch die folgenden vier Winkel ausgeglichen werden.

$$
\begin{array}{rlr}
\text { I. } & n_{9} y=86^{\circ} & 44,8^{\prime}, \\
\text { II. } & C y=55 & 44,0, \\
\text { III. } & P y=82 & 8,8, \\
\text { IV. } & M y=87 & 17,8 .
\end{array}
$$

Man bestimmt aus je zweien von ihnen den Winkel $y M C$ bzw. $y M P$, die sich nur um den festgelegten Winkel $C M P$ unterscheiden. Man benützt das Dreieck $n_{3} M y$ mit I und IV (und dem schon festgelegten $n_{3} M$ ), das Dreieck $C y M$ mit II und IV (und $C M=90^{\circ}$ ) und das Dreieck $P y M$ mit III und IV (und dem schon festgelegten $P M$ ) und erhält für den Winkel $y M C$ die drei Werte $55^{\circ} 41,7^{\prime}, 55^{\circ} 11,4^{\prime}$ und $55^{\circ} 42,0^{\prime}$, demnach als Mittel $55^{\circ} 41,7^{\prime}$.

Damit nun $C y$ und $P y$ genau in die Triangulation hineinpassen, müssen sie den veränderten Winkeln $y M C$ und $y M P$ entsprechend etwas abgeåndert werden. Diese Änderungen der Seiten sind in praxi ebenso groß wie die Änderungen der gegenüberliegenden Winkel, weil die drei Seiten von $M$ aus, nämlich $M y, M C$ und $M P$ beinahe $90^{\circ}$ sind. $C y$ wird dadurch zu $35^{\circ} 44,0^{\prime}+0,3^{\prime}=53^{\circ} 44,3^{\prime}$ und $P y$ zu $82^{\circ} 8,8^{\prime}-0,3^{\prime}=82^{\circ} 8,5^{\prime}$ y $M$ habe ich an der Änderung nicht mehr teilnehmen lassen, was bei der Geringfügigkeit der ganzen Ausgleiche wohl erlaubt sein wird. Die Unterschiede gegenüber den gemessenen Werten sind überhaupt sehr gering. Nur die Winkel gegen die Fläche $n$ mußten wegen der Störung von $n$ etwas größere Abänderungen, nämlich $0,5^{\prime}, 1,2^{\prime}$ und $2,3^{\prime}$ erfahren, bleiben also auch hier überall in den Fehlergrenzen. Eine Gegenüberstellung der gemessenen und ausgeglichenen Winkel möge hier folgen.

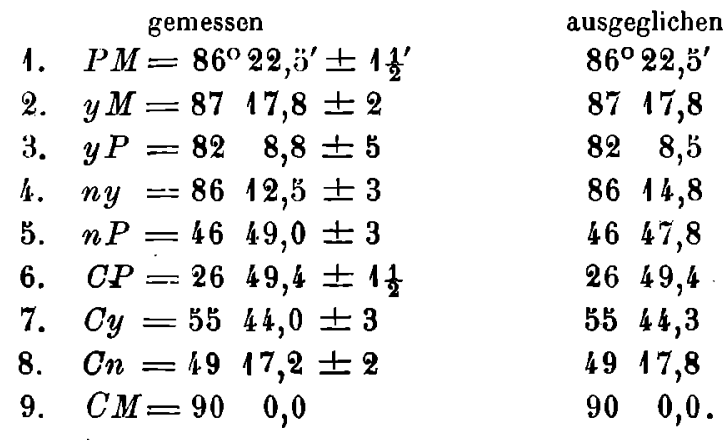


Aus diesen ausgeglichenen Winkeln berechnen sich folgende Konstanten des Albits von Rischuna:

$$
\begin{aligned}
& a: b: c=0,6352: 1: 0,5584, \\
& \alpha=94^{\circ} 14,6^{\prime}, \quad 2 R-A=86^{\circ} 22,5^{\prime}=P M, \\
& \beta=11635,7, \quad 2 R-B=6329,4=P k, \\
& \gamma=8746,0, \quad 2 R-C=9022,6=k M .
\end{aligned}
$$

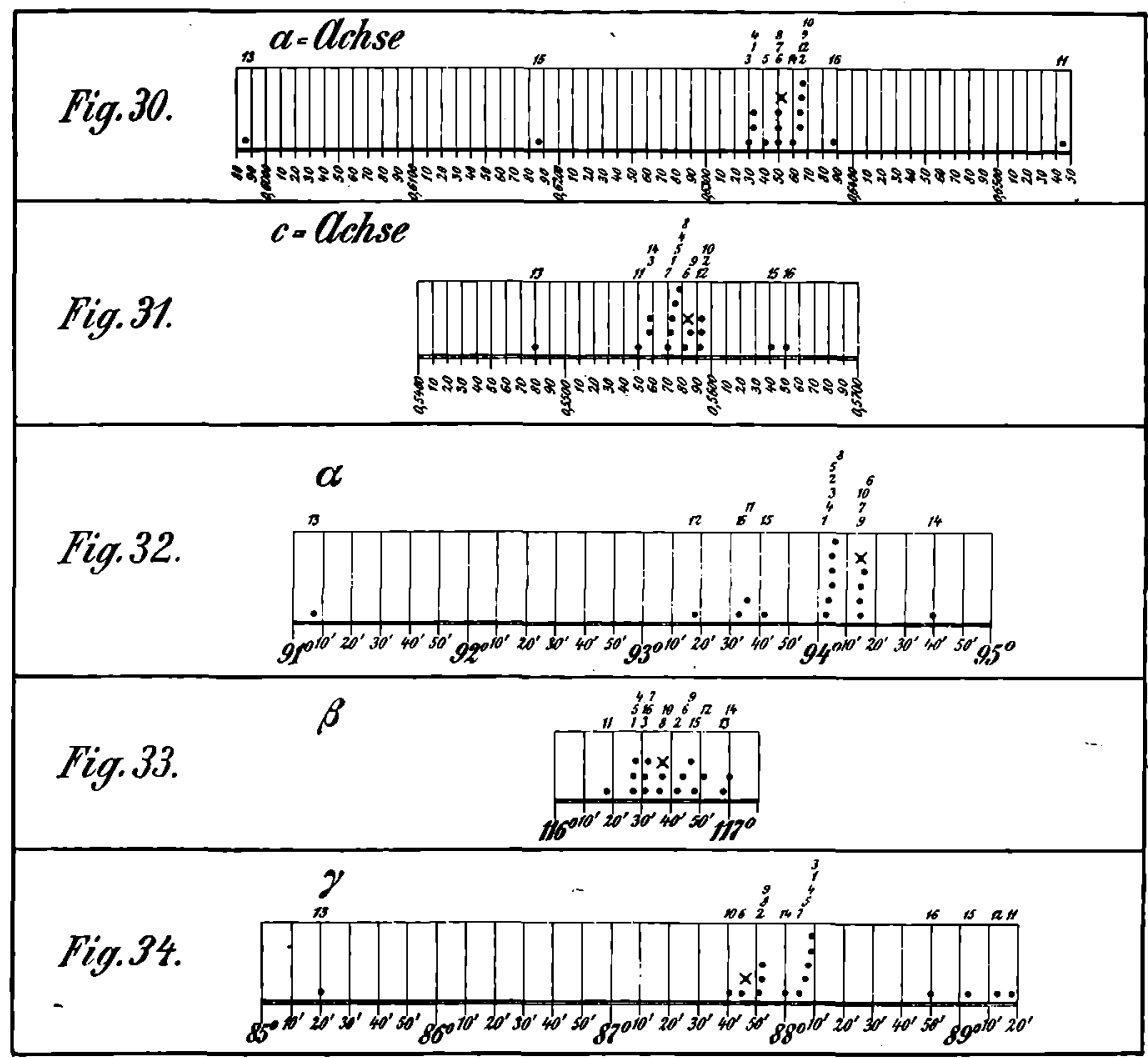

Vergleicht man diese Werte mit den bisher an Albit gefundenen, so kann dies am schnellsten durch die Häufungsbilder geschehen, die E. A.

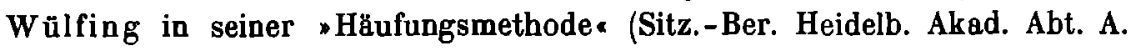
1916, 11. Abh. S. 12) gegeben hat und die hier in den Fig. 30-34 mit Ergänzung der obigen Werte von Rischuna in Form von Kreuzen abgebildet sind. Man sieht, $\operatorname{da} \beta$ die Werte für $a, c, \alpha$ und $\beta$ im Haufen der übrigen liegen und nur $\gamma$ sich etwas seitlich befindet. Der Winkel $\gamma$ wird aber besonders stark durch die Messungen in der Prismenzone beeinflußt, 
die wohl bei allen Albiten dieselbe Unruhe zeigen. Öfters sind diese Prismenwinkel herangezogen worden, wie auch ich dies anfangs beim RischunaAlbit wegen der scheinbar vorzüglichen Ausbildung der Prismenflåchen zu tun geneigt war.

Eine Gewăhr für die sichere Festlegung des Achsenkreuzes des Albits von Rischuna bietet einerseits die Umgehung der Prismenflächen und sonstiger verhältnismāßig schlecht oder seltener gut ausgebildeten Flächen, andererseits die Anwendung der Häufungsmethode, die gerade bei einem so unruhig gewachsenen Mineral wie Albit die Fülle der Beobachtungen zu übersehen und zu sichten erlaubt. 